\section{Contact points between lexical retrieval and sentence production}

\author{
Gabriella Vigliocco ${ }^{\mathrm{a}}$ and Marco Zorzi ${ }^{\mathrm{b}}$ \\ ${ }^{a}$ Department of Psychology, University of Wisconsin-Madison, Madison, WI \\ 53706; 'b Department of Psychology, University of Trieste, 34123 Trieste, Italy. \\ gviglioc@facstaff.wisc.edu \\ psych.wisc.edu/faculty/pages/gvigliocco/GV.html \\ zorzi@uts.univ.trieste.it \\ www.psychol.ucl.ac.uk/marco.zorzi/marco.html
}

\begin{abstract}
Speakers retrieve words to use them in sentences. Errors in incorporating words into sentential frames are revealing with respect to the lexical units as well as the lexical retrieval mechanism; hence they constrain theories of lexical access. We present a reanalysis of a corpus of spontaneously occurring lexical exchange errors that highlights the contact points between lexical and sentential processes.
\end{abstract}

We retrieve words in order to communicate and we communicate using sentences, not (or very rarely) words in isolation. The lexical retrieval mechanism and sentence building machinery need to be coordinated. Errors in assigning lexical entries in a sentential frame can be revealing with respect to the lexicalization process and vice versa: errors in lexical retrieval may suggest ways in which sentence construction is controlled by lexical structures.

Levelt, Roelofs \& Meyer discuss how lexical retrieval affects sentence construction (sect. 5.4); however, they do not draw the corresponding implications with respect to how sentence construction constrains lexical retrieval. The danger of this approach is explaining phenomena that are paradigmatically related to sentence level processes solely in terms of lexical properties. The goal of this commentary is to fill this gap, providing constraints for Levelt et al.'s theory on the basis of observations of slips of the tongue. We present a reanalysis of a corpus of speech errors in Spanish (del Viso et al. 1987) focusing on lexical exchanges. An example of an exchange error in English (from the MIT corpus; Garrett 1980) is reported below.

Error: How many pies does it take to make an apple?

Intended: How many apples does it take to make a pie?

In the example, "apple" and "pie" swapped position. The plural marking on the target "apple" does not move with the lexical stem; it is stranded. These errors reflect misassignment of lexical elements into sentential frames.

The rationale for considering Spanish is that Spanish is a highly inflected language that allows us to better assess the morphological status of the exchanged units. Exchange errors come in five different flavors in the Spanish corpus (total number of errors considered $=134$ ). Examples in the different categories are provided in Table 1. Errors, such as those in categories 1-4, involve nouns; our argument is built around what happens to features shared by the nouns and the determiners (such as number and gender) when the exchange occurs. Errors in category 5 instead involve units from different grammatical categories (more precisely an adjective and an adverb). Let us consider the units involved in the different exchanges.

In phrasal exchanges, both the nouns and the determiners (i.e., the whole noun phrases) move together. In word exchanges, the nouns with their bound inflectional morphology exchange, leaving behind the determiners fully inflected for the targets. In stem exchanges, only the word stems move, leaving behind bound inflections (in the example, "number" does not move with the lexical stem). Ambiguous exchanges could be described as phrasal, word, or stem exchanges. In examples such as the reported one, the two nouns share number and gender, making a more precise classification impossible. Finally, while for phrasal, word, and stem exchanges the two exchanging elements share the same grammatical category and belong to separate syntactic phrases, in morpheme exchanges the two units do not share grammatical cat-

Table 1 (Vigliocco \& Zorzi). Examples of different exchange errors in Spanish

\begin{tabular}{|c|c|}
\hline Error & Target \\
\hline \multicolumn{2}{|l|}{ 1. Phrasal Exchange } \\
\hline . . . las chicas de la cara estan ... & .. . la cara de las chicas esta ... \\
\hline (the-F,P girl-F,P of the-F,S face-F,S are) & (the-F,S face-F,S of the-F,P girl-F,P is) \\
\hline \multicolumn{2}{|l|}{ 2. Word Exchange } \\
\hline . . . la corte del imagen Ingles & . . . la imagen del corte ingles \\
\hline (the-F,S cut-M,S of the-M,S image-F,S English) & (the-F,S image-F,S of the-M,S cut-M,S English) \\
\hline \multicolumn{2}{|l|}{ 3. Stem Exchange } \\
\hline Pasame las tortillas para la patata & ... las patatas para la tortilla \\
\hline (Pass me the-F,P omelette-F,P for the-F,S potato-F,S) & (. . . the-F,P potato-F,P for the-F,S omelette-F,S) \\
\hline \multicolumn{2}{|l|}{ 4. Ambiguous } \\
\hline . . . le han dedicado periodicos sus editoriales & . . . le han dedicado editoriales sus periodicos \\
\hline (to-her have devoted periodicals- $\mathrm{M}, \mathrm{P}$ on editorials-M,P) & ( . . . editorials- $\mathrm{M}, \mathrm{P}$ on periodicals- $\mathrm{M}, \mathrm{P})$ \\
\hline \multicolumn{2}{|l|}{ 5. Morpheme Exchange } \\
\hline . . . un efecto significativamente estadistico & . . . un efecto estadisticamente significativo \\
\hline (an-M,S effect-M,S significantly statisticant-M,S) & (an-M,S effect-M,S statistically significant-M,S) \\
\hline
\end{tabular}

Note: In the English translations, $\mathrm{F}=$ feminine; $\mathrm{M}=$ masculine; $\mathrm{S}=$ singular; $\mathrm{P}=$ plural. 
egory ("estadisticamente" is an adverb; "significativo" is an adjective) and are in the same phrase. Note that stem and morpheme exchanges involve the same units (a word stem) but differ in terms of their syntactic environment.

There were 134 relevant errors in the corpus. Of these, 21 were phrasal exchanges; 1 was a word exchange; 41 were stem exchanges; 49 were ambiguous cases; and finally 22 were morpheme exchanges.

A very important observation is that word exchanges are virtually absent. The example reported above is the only one we found in the corpus. This suggests that fully inflected words are not lexical units of encoding.

What are the implications of these observations for the model of lexical retrieval proposed by Levelt et al.? In their discussion of exchange errors, Levelt et al. (sect. 5.4.5) assume a distinction between word and morpheme exchanges and attribute the first to lemma level and the second to word form level. We agree that errors reflect both lemma and word form level representations. Furthermore, we agree with their analysis of morpheme exchanges. However, Levelt et al. conflate under "word exchanges" three types of errors: phrasal, word, and stem exchanges. We believe it is important to separate them.

Both phrasal and stem exchanges involve lemmas, but they occur during two different processes. Phrasal exchanges would arise because of a misassignment of grammatical functions to lemmas. In the example in Table 1, "cara" and "chica" are "subject" and "modifier," respectively, in the target utterance, but their functions are swapped in the error (for a similar treatment see Bock \& Levelt 1994). Hence these errors reflect the information flow from a "message level" sentential representation to lemmas. Stem exchanges, instead, would reflect a mistaken insertion of lemmas into frames that specify inflectional morphology.

The contrast between phrasal and stem exchanges suggests a system in which grammatical functions are first assigned to lemmas. Next, a syntactic frame would be initiated on the basis of these functions. The frame would specify inflectional morphology on the basis of the message (for conceptual features such as number) as well as on the basis of the specific lemmas (for lexical features such as gender). ${ }^{1}$ Lemmas would finally be inserted in these frames. Hence features such as number and gender for nouns would be specified during the construction of the corresponding noun phrases, not at a lexical level. The absence of true word exchanges in the corpus strengthens this hypothesis. If these features were specified at a lexical level, we should have observed a larger number of word exchanges. In this account, inflections would be assigned to a developing syntactic frame when lemmas are selected and "tagged" for grammatical functions before stem exchanges occur, not after as Levelt et al. argue.

To sum up, our analysis highlights further important sentential constraints on lexical retrieval that Levelt et al. need to take into account to model lexical retrieval during connected speech.

\section{ACKNOWLEDGMENT}

Support was provided by a NSF grant (SBR 9729118) to the first author. We would like to thank Ines Anton-Mendes, Merrill Garrett, Rob Hartsuiker, and Jenny Saffran for their comments.

NOTE

1. The picture may actually be more complex, and conceptually motivated features may be treated differently from lexically motivated features. Interestingly, there are a few $(=3)$ cases in the corpus when number is stranded while grammatical gender moves with the word (and the determiners agree with the gender of the error, not the target). However, a substantially larger number of cases would be necessary to see whether conceptual and lexical features behave differently. 